九州大学学術情報リポジトリ

Kyushu University Institutional Repository

\title{
Histogram Interpolation Methods for Image Contrast Enhancement
}

Yu, Hengjun

Department of Communication Design Science, Kyushu University

Inoue, Kohe $\mathrm{i}$

Department of Communication Design Science, Kyushu University

Hara, Kenji

Department of Communication Design Science, Kyushu University

Urahama, Ki ichi

Department of Communication Design Science, Kyushu University

ht tp://hdl. hand le. net/2324/1806139

出版情報: Journal of the Institute of Industrial Applications Engineers. 5 (1), pp.7-14, 201701-25. 産業応用工学会

バージョン:

権利関係 : 


\title{
Paper
}

\section{Histogram Interpolation Methods for Image Contrast Enhancement}

\author{
HenguUn Yu* Member, Kohei InOUE* Member \\ KenJI HARA* Non-member, KIICHI URAHAMA* Member
}

(Received October 1, 2015, revised December 1, 2015)

\begin{abstract}
Histogram equalization (HE) is a well-known technique for image contrast enhancement. However, $\mathrm{HE}$ frequently over-enhances the contrast of a given image to produce false contours in the enhanced image. One of the reasons for the production of false contours is the sparseness of the equalized histogram, which means that there exist gaps in continuous-tone. In this paper, we propose three methods for interpolating the sparse histogram obtained by HE, and utilize the interpolated histograms as the target ones for histogram specification or histogram matching, which transforms the histogram of a given image into the specified target histogram. Filling the gaps in equalized histogram by interpolation, we can reduce the occurrence of false contours. Experimental results show that the proposed methods can alleviate the over-enhancement of the contrast, which occurs frequently when we use HE. We also evaluate the quality of contrast-enhanced images by using three image quality measures, where the proposed methods achieve better performance than HE in many cases.
\end{abstract}

Keywords: Contrast enhancement, Histogram equalization, Histogram specification, Histogram interpolation, Image quality measures

\section{Introduction}

Contrast enhancement (CE) is an important issue in the field of image processing. Histogram equalization (HE) [1] is a well-known method for CE. However, HE tends to overenhance the contrast of low contrast images, and amplify noise [2]. As a result, the enhanced images may have false contours. We can find the footprint of the over-enhancement in the histogram of the histogram-equalized image, i.e., the resultant histogram frequently becomes sparse, in other words, a number of elements of the histogram are zero. The sparsity of the histogram indicates that there exist gaps in continuous-tone.

To overcome such a difficulty in HE, we have recently proposed a histogram specification (HS) method [3] based on the fast ordering algorithm by Nikolova and Steidl [4], which bridges the gap by filling the empty bins in the equalized histogram with piecewise constant non-zero values. This HS method achieved better performance than conventional HE for most of the tested images. However, the HS method is computationally expensive compared to HE because Nikolova's method [4] is a demanding procedure. The CPU time of the HS method was eight times larger than HE in our previous experiment [3].

To reduce the computational costs in the HS method [3], we propose computationally efficient methods for appropriate image contrast enhancement without Nikolova's method [4] in this paper. First, we compute the histogram of a histogram-equalized image, and then interpolate it with

* Department of Communication Design Science, Kyushu University

4-9-1, Shiobaru, Minami-ku, Fukuoka 815-8540, Japan

(k-inoue@design.kyushu-u.ac.jp) one of the three proposed interpolation methods: piecewiseconstant, -linear and local min-max interpolations. After that, we use the interpolated histogram as a target histogram for histogram specification method. Experimental results show that the proposed method can suppress the contrast over-enhancement, and attain good performance of image contrast enhancement.

The rest of this paper is organized as follows. Section 2 briefly summarizes HE. Section 3 proposes three histogram interpolation methods. Section 4 describes histogram specification method using the interpolated histogram as the target one. Section 5 describes image quality measures used in our experiments. Section 6 shows experimental results. Finally, Section 7 concludes this paper.

\section{Histogram Equalization}

Let $G$ be a grayscale image, and let $g_{i j}$ be the intensity at the position $(i, j)$ of a pixel in $G$ for $i=1,2, \ldots, m$ and $j=$ $1,2, \ldots, n$, where $g_{i j} \in \mathcal{L}=\{0,1, \ldots, L-1\}$, and $L$ denotes the number of intensity levels, e.g., $L=2^{8}=256$ for 8 -bit images. Let $\boldsymbol{h}=\left[h_{0}, h_{1}, \ldots, h_{L-1}\right]$ be the histogram of $G$, then the $k$ th element of $\boldsymbol{h}$ is given by $h_{k}=\sum_{i=1}^{m} \sum_{j=1}^{n} \delta_{g_{i j}, k}$ where $\delta_{g_{i j}, k}$ denotes the Kronecker delta such that $\delta_{g_{i j}, k}=1$ if $g_{i j}=k, \delta_{g_{i j}, k}=0$ otherwise. Let $\boldsymbol{H}=\left[H_{0}, H_{1}, \ldots, H_{L-1}\right]$ be the cumulative histogram of $\boldsymbol{h}$, where the $k$ th element of $\boldsymbol{H}$ is given by $H_{k}=\sum_{k^{\prime}=0}^{k} h_{k^{\prime}}$. Then the histogram-equalized image $G^{E}=\left[g_{i j}^{E}\right]$ of $G$ is given by

$$
g_{i j}^{E}=\operatorname{round}\left(a H_{g_{i j}}+b\right) \text {, }
$$


where 'round' means to round a given argument toward the nearest integer, and $a$ and $b$ are given by

$$
a=\frac{L-1}{m n}, \quad b=0
$$

or

$$
a=\frac{L-1}{m n-H_{g_{\min }}}, \quad b=-a H_{g_{\min }},
$$

where $g_{\min }=\min \left\{g_{i j} \in G\right\}$. The intensity transformation in (1) with (2) or (3) guarantees that $g_{i j}^{E} \in \mathcal{L}$.

Let $\phi^{E}$ be the intensity transformation in (1):

$$
\phi^{E}(k)=\operatorname{round}\left(a H_{k}+b\right) \text { for } k \in \mathcal{L} \text {. }
$$

Then the $l$ th element of the histogram $\boldsymbol{h}^{E}$ of $G^{E}$ is given by

$$
h_{l}^{E}= \begin{cases}0, & \text { if } l \neq \phi^{E}(k) \text { for all } k \in \mathcal{L}, \\ \sum_{k: l=\phi^{E}(k)} h_{k}, & \text { otherwise, }\end{cases}
$$

where $\sum_{k: l=\phi^{E}(k)}$ means to sum up the values with the indices $k$ satisfying $l=\phi^{E}(k)$. Let $\boldsymbol{H}^{E}$ be the cumulative histogram of $\boldsymbol{h}^{E}$, then the $l$ th element of $\boldsymbol{H}^{E}$ is given by

$$
H_{l}^{E}=\sum_{l^{\prime}=0}^{l} h_{l^{\prime}}^{E}=\sum_{l^{\prime}=0}^{l} \sum_{k: l^{\prime}=\phi^{E}(k)} h_{k} .
$$

Suppose that $k_{1}=\max \left\{k \mid h_{k} \neq 0\right.$ and $\left.\phi^{E}(k)=\phi_{1}\right\}$ and $k_{2}=$ $\max \left\{k \mid h_{k} \neq 0\right.$ and $\left.\phi^{E}(k)=\phi_{2}\right\}$ for $\phi_{1} \in\left\{\phi^{E}(k) \mid k \in \mathcal{L}\right\}$ and $\phi_{2} \in\left\{\phi^{E}(k) \mid k \in \mathcal{L}\right\}$ such that $\phi_{1}<\phi_{2}$. Then the derivative of $\boldsymbol{H}^{E}$ can be approximated by

$$
\begin{aligned}
\frac{H_{\phi^{E}\left(k_{2}\right)}^{E}-H_{\phi^{E}\left(k_{1}\right)}^{E}}{\phi^{E}\left(k_{2}\right)-\phi^{E}\left(k_{1}\right)} & =\frac{\sum_{l=0}^{\phi^{E}\left(k_{2}\right)} \sum_{k: l=\phi^{E}(k)} h_{k}-\sum_{l^{\prime}=0}^{\phi^{E}\left(k_{1}\right)} \sum_{k^{\prime}: l^{\prime}=\phi^{E}\left(k^{\prime}\right)} h_{k^{\prime}}}{\operatorname{round}\left(a H_{k_{2}}+b\right)-\operatorname{round}\left(a H_{k_{1}}+b\right)} \\
& \approx \frac{\sum_{k=0}^{k_{2}} h_{k}-\sum_{k^{\prime}=0}^{k_{1}} h_{k^{\prime}}}{\left(a H_{k_{2}}+b\right)-\left(a H_{k_{1}}+b\right)} \\
& =\frac{H_{k_{2}}-H_{k_{1}}}{a\left(H_{k_{2}}-H_{k_{1}}\right)}=\frac{1}{a} .
\end{aligned}
$$

Hence, the slope of $\boldsymbol{H}^{E}$ is approximately constant similar to that of the cumulative histogram of strictly equalized histogram.

In general, the intensity transformation in (4) is a manyto-one mapping, and as long as it is not a one-to-one mapping, $\boldsymbol{h}^{E}$ includes 0 as described in (5). Therefore, the histogram obtained by histogram equalization frequently become sparse.

\section{Histogram Interpolation}

In the above section, we showed that the equalized histogram $\boldsymbol{h}^{E}$ frequently includes 0 elements. The successive occurrence of 0 elements in $\boldsymbol{h}^{E}$ can lead to discontinuous tone which may cause false contours in the histogramequalized image $G^{E}$. In this section, we propose three histogram interpolation methods for replacing the 0 elements with positive values: piecewise-constant, piecewise-linear and local min-max interpolations, which will be described in the following subsections.
3.1 Piecewise-Constant Interpolation Let $\boldsymbol{h}^{P C}=$ $\left[h_{0}^{P C}, h_{1}^{P C}, \ldots, h_{L-1}^{P C}\right]$ be an interpolated histogram which is initialized by $\boldsymbol{h}^{E}: \boldsymbol{h}^{P C}=\boldsymbol{h}^{E}$. Then, for $l=L-2, L-3, \ldots, 0$ in this order, we update the values of $h_{l}^{P C}$ as follows:

$$
h_{l}^{P C}= \begin{cases}h_{l+1}^{P C}, & \text { if } h_{l}^{P C}=0 \\ h_{l}^{P C}, & \text { otherwise. }\end{cases}
$$

The resultant $\boldsymbol{h}^{P C}$ becomes piecewise-constant histogram because a non-zero element $h_{l+1}^{P C}$ is repeatedly copied to successive 0 elements neighboring to the left side.

3.2 Piecewise-Linear Interpolation Let $\boldsymbol{h}^{P L}=$ $\left[h_{0}^{P L}, h_{1}^{P L}, \ldots, h_{L-1}^{P L}\right]$ be the resultant histogram of piecewiselinear interpolation for $\boldsymbol{h}^{E}$. Then the $l$ th element $h_{l}^{P L}$ is given by

$$
h_{l}^{P L}= \begin{cases}\frac{\left(l^{R T}-l\right) h_{l^{L T}}^{E}+\left(l-l^{L T}\right) h_{l^{R T}}^{E},}{l^{R T}-l^{L T}}, & \text { if } h_{l}^{E}=0, \\ h_{l}^{E}, & \text { otherwise }\end{cases}
$$

where

$$
\begin{aligned}
& l^{L T}=\max _{l^{\prime}}\left\{l^{\prime} \mid l^{\prime}<l, h_{l^{\prime}}^{E} \neq 0\right\}, \\
& l^{R T}=\min _{l^{\prime}}\left\{l^{\prime} \mid l^{\prime}>l, h_{l^{\prime}}^{E} \neq 0\right\} .
\end{aligned}
$$

3.3 Local Min-Max Interpolation In many cases, the above two methods work well. However, if there are big gaps and impulsive elements in the equalized histogram $\boldsymbol{h}^{E}$, then the two methods can change the shape of histogram largely from that of $\boldsymbol{h}^{E}$. As a result, the resultant image may be contradictory to the original image. We found such examples in our experiments. For those images, in this subsection, we propose a local min-max interpolation method as follows.

Let $\boldsymbol{h}^{M M}=\left[h_{0}^{M M}, h_{1}^{M M}, \ldots, h_{L-1}^{M M}\right]$ be the resultant histogram of local min-max interpolation for $\boldsymbol{h}^{E}$. Then we first compute an intermediate histogram $\boldsymbol{h}^{U}=\left[h_{0}^{U}, h_{1}^{U}, \ldots, h_{L-1}^{U}\right]$ of which the $l$ th element $h_{l}^{U}$ is given by

$$
h_{l}^{U}=\max \left\{h_{l-1}^{E}, h_{l}^{E}, h_{l+1}^{E}\right\},
$$

where we define that $h_{-1}^{E}=h_{L}^{E}=-\infty$. Next, we compute $\boldsymbol{h}^{M M}$ of which the $l$ th element $h_{l}^{M M}$ is given by

$$
h_{l}^{M M}=\min \left\{h_{l-1}^{U}, h_{l}^{U}, h_{l+1}^{U}\right\},
$$

where we define that $h_{-1}^{U}=h_{L}^{U}=\infty$.

The above histogram modification is local because (12) and (13) look both adjacent elements only. Therefore, this modification preserves the original shape of histogram better than piecewise-constant and piecewise-linear interpolations.

\section{Histogram Specification}

In this section, we describe the procedure of histogram specification using the above interpolated histograms as the target histograms. 
Let $\boldsymbol{h}^{X}=\left[h_{0}^{X}, h_{1}^{X}, \ldots, h_{L-1}^{X}\right]$ be one of the above three interpolated histograms, i.e., $X \in\{P C, P L, M M\}$, and let $\boldsymbol{H}^{X}=\left[H_{0}^{X}, H_{1}^{X}, \ldots, H_{L-1}^{X}\right]$ be the cumulative histogram of $\boldsymbol{h}^{X}$. Then the $l$ th element $H_{l}^{X}$ of $\boldsymbol{H}^{X}$ is given as $H_{l}^{X}=$ $\sum_{l^{\prime}=0}^{l} h_{l^{\prime}}^{X}$. Let $G^{X}=\left[g_{i j}^{X}\right]$ be the output image of the histogram specification with $\boldsymbol{h}^{X}$. Then the pixel values of $G^{X}$ are given by

$$
g_{i j}^{X}=\arg \min _{l}\left\{\left|H_{L-1} H_{l}^{X}-H_{L-1}^{X} H_{g_{i j}}\right|\right\}
$$

for $i=1,2, \ldots, m$ and $j=1,2, \ldots, n$.

\section{Image Quality Measures}

In this section, we briefly summarize three image quality measures used in our experiments.

5.1 Expected Measure of Enhancement by Gradient Expected measure of enhancement by gradient (EMEG) proposed by Celik [5] is defined by

$$
\operatorname{EMEG}(G)=\frac{1}{M N} \sum_{I=1}^{M} \sum_{J=1}^{N} \frac{1}{\beta} \max \left(\frac{G_{I, J}^{d x, h}}{G_{I, J}^{d x, l}+\epsilon}, \frac{G_{I, J}^{d y, h}}{G_{I, J}^{d y, l}+\epsilon}\right),
$$

where $G_{I, J}^{d x, h}\left(G_{I, J}^{d y, h}\right)$ and $G_{I, J}^{d x, l}\left(G_{I, J}^{d y, l}\right)$ are the maximum and minimum values of absolute valued derivatives in horizontal (vertical) direction of $w_{1} \times w_{2}$ sub-block $G_{I, J}$ of an image $G, \beta=L-1$, and $\epsilon$ is a positive constant. We set $\epsilon=$ $1, w_{1}=w_{2}=8, M=\operatorname{fix}\left(m / w_{1}\right)$ and $N=\operatorname{fix}\left(n / w_{2}\right)$ where fix denotes a function for rounding toward zero. The larger EMEG is, the higher the image quality is.

\subsection{Gradient Magnitude Similarity Deviation Gra-} dient magnitude similarity deviation (GMSD) proposed by Xue et al. [6] is defined by

$$
\operatorname{GMSD}\left(G, G^{\prime}\right)=\sqrt{\frac{1}{m n} \sum_{i=1}^{m} \sum_{j=1}^{n}\left(\mathrm{GMS}_{i j}^{G, G^{\prime}}-\mathrm{GMSM}^{G, G^{\prime}}\right)^{2}},
$$

where $\mathrm{GMSM}^{G, G^{\prime}}$ denotes the average of $\mathrm{GMS}_{i j}^{G, G^{\prime}}$ which is the value of GMS map at $(i, j)$ defined by

$$
\mathrm{GMS}_{i j}^{G, G^{\prime}}=\frac{2 m_{G, i j} m_{G^{\prime}, i j}+c}{m_{G, i j}^{2} m_{G^{\prime}, i j}^{2}+c},
$$

where $m_{G, i j}$ and $m_{G^{\prime}, i j}$ denote the gradient magnitudes of $G$ and $G^{\prime}$ at $(i, j)$, respectively, and $c$ is a positive constant for numerical stability. The lower value of GMSD means higher image quality.

5.3 EMEG over GMSD EMEG over GMSD proposed by Yu et al. [3] is defined by

$$
\mathrm{E} / \mathrm{G}\left(G, G^{\prime}\right)=\frac{\operatorname{EMEG}\left(G^{\prime}\right)}{\operatorname{GMSD}\left(G, G^{\prime}\right)} .
$$

The larger $\mathrm{E} / \mathrm{G}$ is, the higher the image quality is.

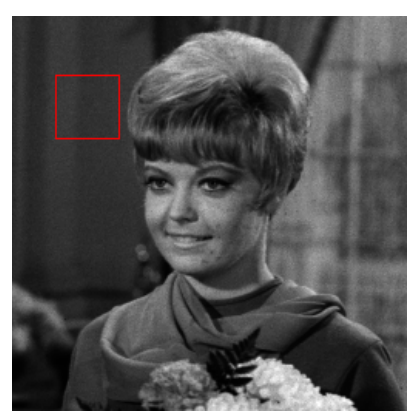

(a) Original (Girl)

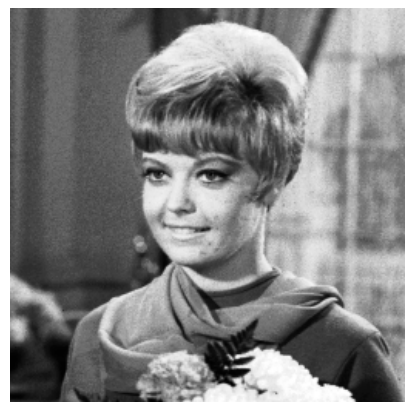

(c) Piecewise-const.

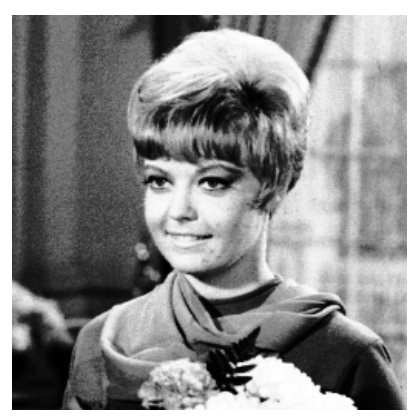

(b) $\mathrm{HE}$

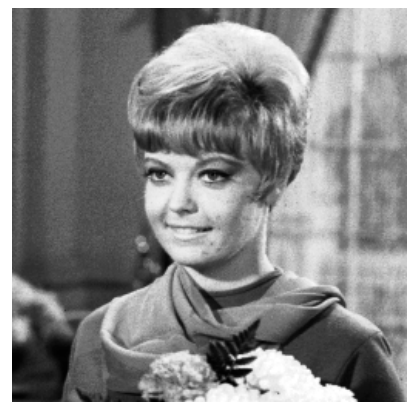

(d) Piecewise-linear
Figure 1: Example of image contrast enhancement.

\section{Experimental Results}

In this section, we show experimental results with grayscale images in the SIDBA standard image database [7]. First, we show an example of contrast over-enhancement by HE and its suppression by the proposed piecewise-constant and linear interpolation methods. Next, we show another example of contrast over-enhancement still existing after the application of the piecewise-constant and -linear interpolation methods and its suppression by the local min-max interpolation method. After that, we will show the results of quantitative evaluation of the quality of enhanced images based on image quality measures described in the above section.

6.1 Suppressing Over-Enhancement in HE Fig. 1 shows an example of contrast enhancement, where we selected a grayscale image of Fig. 1(a) as an input image from the SIDBA standard image database [7]. Fig. 1(b) shows the result of histogram equalization (HE), which enhances the contrast excessively. For example, the zoomed regions of wall behind the girl (the region is surrounded by a red frame in Fig. 1(a)) corresponding to the images in Fig. 1 are shown in Fig. 2, where random noise is enhanced by false contouring effect. Figs. 1(c) and (d) show the results of the proposed piecewise-constant and -linear interpolations, respectively, both of which suppress the contrast over-enhancement caused by HE. The corresponding zoomed parts are shown in Figs. 2(c) and (d), where the random noise is not so noticeable as it in Fig. 2(b).

Figs. 3(a) and (b) show the histograms of Figs. 1(a) and (b), respectively, where the vertical and horizontal axes denote the number of pixels and the intensity, respectively. Fig. 3(b) given by HE is not equalized in a strict meaning, but the cumulative histogram is approximately linearized or the slope of the cumulative histogram is approximately con- 


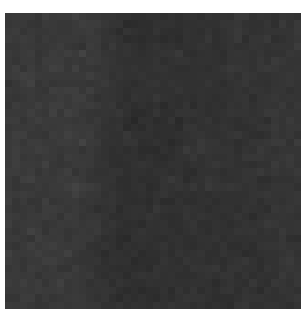

(a) Original

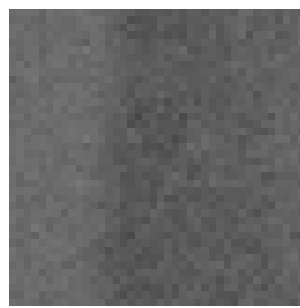

(c) Piecewise-const.

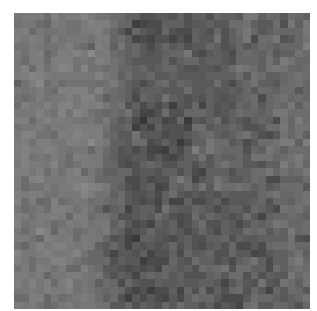

(b) $\mathrm{HE}$

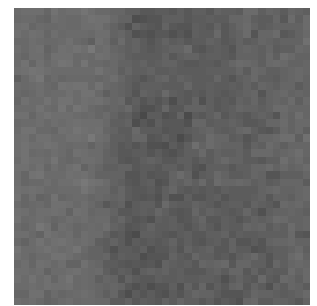

(d) Piecewise-linear
Figure 2: Zoomed partial images.

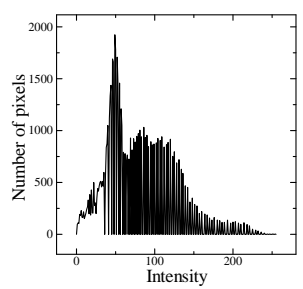

(a) Original

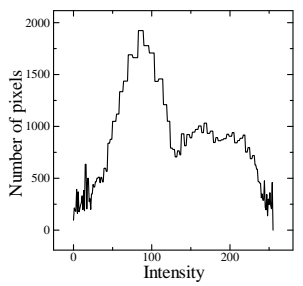

(c) Piecewise-const.



(b) $\mathrm{HE}$

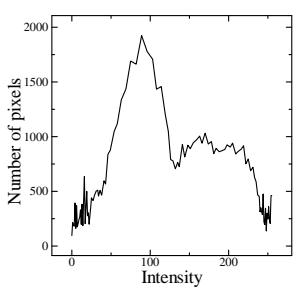

(d) Piecewise-linear
Figure 3: Histograms.

stant as shown in Section 2. Fig. 3(b) has many bins of 0, the existence of which means that there are gaps in continuoustone in Fig. 1(b). Figs. 3(c) and (d) show the interpolated histograms of $\boldsymbol{h}^{E}$ in Fig. 3(b) by the piecewise-constant and -linear interpolation methods, respectively. In the interpolated histograms, 0 -valued bins are filled with non-zero values by piecewise-constant and -linear interpolations. These histograms are used for target ones in the histogram specification method described in Section 4, and the resultant images are shown in Figs. 1(c) and (d).

6.2 Suppressing Over-Enhancement in PiecewiseConstant and -Linear Interpolations The above piecewise-constant and -linear interpolation methods can alleviate the over-enhancement of contrast in HE well in many cases. However, in our experiments, we found some exceptions, for which the two methods still over-enhanced the contrast, and we applied the local min-max interpolation method to such images to suppress the over-enhancement.

Fig. 4 shows such an example, where Figs. 4(a) and (b)

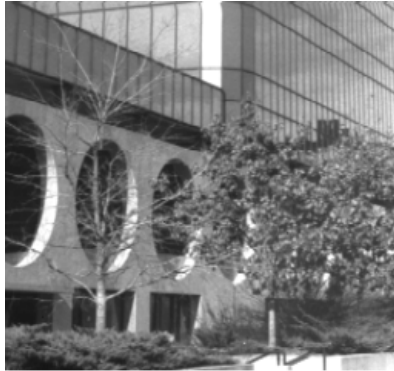

(a) Original (Building)

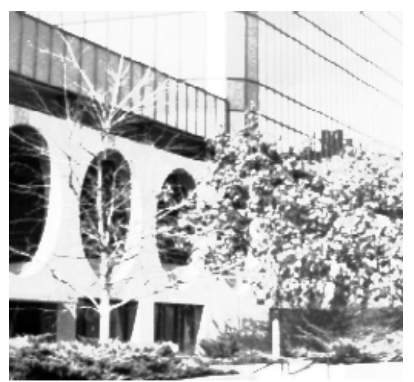

(c) Piecewise-const.



(b) $\mathrm{HE}$

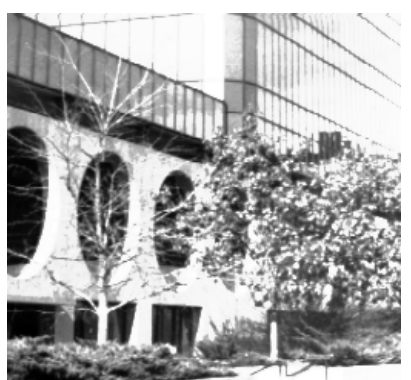

(d) Piecewise-linear

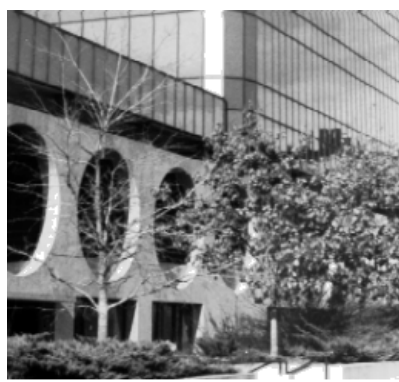

(e) Local min-max

Figure 4: Example of image contrast enhancement.

show the original image and the resultant image of HE, which enhances the contrast well. On the other hand, the piecewise-constant and -linear interpolation methods enhance the contrast excessively as shown in Figs. 4(c) and (d), respectively, where we can see the intensity saturation on the holey wall of the building and the leaves of trees. Fig. 4(e) shows the result of the proposed local min-max interpolation method, where the over-enhancement is avoided successfully, and the contrast is improved compared with Fig. 4(b), e.g., the inside of the first floor of the building can be seen in Fig.4(e) better than Fig. 4(b).

Fig. 5 shows the histograms of the images in Fig. 4. Fig. 5(a) shows the original histogram which has a big impulsive element at 255 on the horizontal axis. Fig. 5(b) shows the histogram given by $\mathrm{HE}$ which generates a big gap or 0 -valued bins between 237 and 254 on the horizontal axis, and also has the impulsive element as well as Fig. 5(a). The results of histogram interpolation for Fig. 5(b) are shown in Fig. 6, where the equalized histograms are denoted by green lines, and the interpolated histograms are denoted by red lines. The piecewise-constant and -linear interpolations fill the gap between 237 and 254 with large values as 


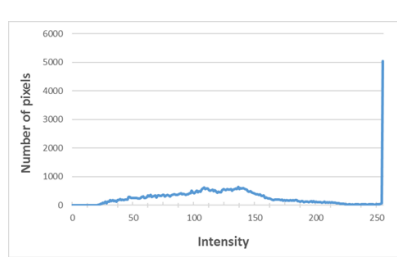

(a) Original

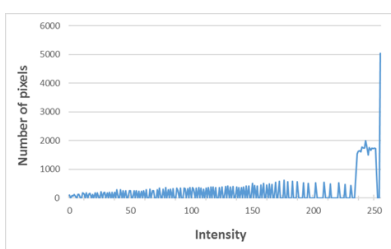

(c) Piecewise-const.

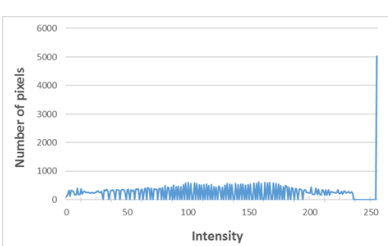

(b) $\mathrm{HE}$

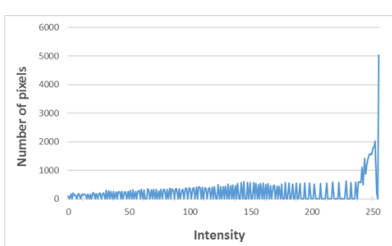

(d) Piecewise-linear

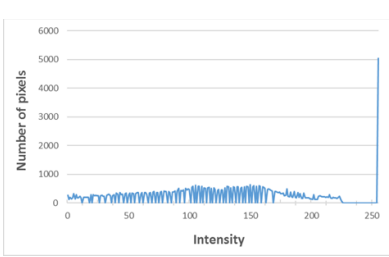

(e) Local min-max

Figure 5: Histograms.

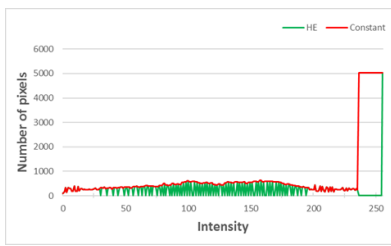

(a) Piecewise-const.

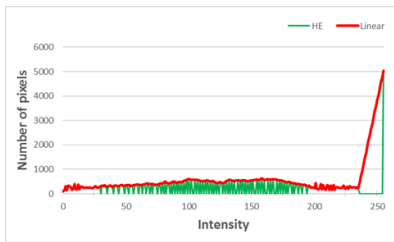

(b) Piecewise-linear

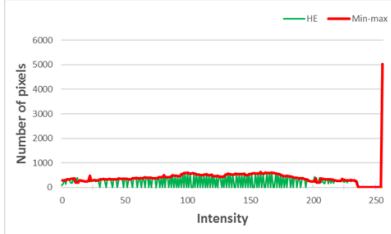

(c) Local min-max

Figure 6: Histogram interpolation.

shown in Figs. 6(a) and (b), respectively. As a result, the shapes of histograms are largely altered by those two methods. On the other hand, as we can see in Fig. 6(c), the local min-max interpolation preserves the gap after the interpolation procedure. The resultant histograms after the histogram specification with the target interpolated histograms in Figs. 6(a)-(c) are shown in Figs. 5(c)-(e), respectively, where Figs. 5(c) and (d) have relatively large values in the interval between 237 and 254 where the gap existed in the equalized histogram (Fig. 3(b)), that indicates the occurrence of the intensity saturation in Figs. 4(c) and (d). On the other hand, Fig. 5(e) preserves the gap, that indicates that the local min-max interpolation method can avoid the intensity saturation or the contrast over-enhancement caused by the piecewise-constant and -linear interpolations.

Table 1 shows the CPU time for the compared four meth-
Table 1: CPU time (ms).

\begin{tabular}{cccc}
\hline HE & Piece.-const. & Piece.-linear & Local min-max \\
\hline 41 & 42 & 41 & 41 \\
\hline
\end{tabular}

ods, which spent almost the same time. Therefore, the proposed methods are computationally efficient as well as HE is. In Table 1, each value is an average value over the twelve images for processing a $256 \times 256$ pixel image. We performed the experiments using $\mathrm{C}++$ and Visual Studio 2015 IDE on an Intel Core i5-4590 CPU@3.30GHz machine.

6.3 Image Quality Evaluation In this subsection, we show the results of the quantitative evaluation of image quality by using three image quality measures described in Section 5.

Fig. 7 shows the results of image contrast enhancement with the SIDBA standard image database [7]. The first row in Fig. 7 shows the original images, which are enhanced by $\mathrm{HE}$ as shown in the second row, where some images are enhanced excessively, for example, the wall of cabin in image 'Boat' become difficult to see the details. The third and fourth rows show the results of the piecewise-constant and linear interpolations, which suppress the over-enhancement observed in most of the images in the second row, for example, the contrast deterioration in 'Boat' of the second row is avoided in the third and fourth rows. However, 'Lighthouse' is over-enhanced in the third and fourth rows similarly to the example of 'Building' in Fig. 4. The lowest fifth row shows the results of the local min-max interpolation, which suppresses the over-enhancement in 'Lighthouse', and give a better result than the other methods.

The above compared methods have both advantages and disadvantages. To compare the performance of these methods, we evaluated the image quality measures, the values of which are collected in Table 2. We enhanced twelve grayscale images in the SIDBA standard image database [7] by four methods: HE, piecewise-constant and -linear, local min-max interpolations, and evaluated the values of three image quality measures: EMEG, GMSD and E/G. The bottom row in Table 2 shows the average values of the twelve images. For each image and each quality measure, the largest value among the four methods is indicated in boldface. For 'Building' and 'Lighthouse' both of which are over-enhanced by the piecewise-constant and -linear interpolations, the local min-max interpolation gets the best GMSD and E/G values as indicated by underlines in Table 2 .

Among the three image quality measures, GMSD and E/G require two images $G$ and $G^{\prime}$, e.g., an original and the processed images, for computing their values as shown in (16) and (18). Therefore, we cannot compute their values before processing a given image. On the other hand, EMEG can be computed for one image $G$ before the processing as shown in (15). Therefore, we can compute the EMEG values for original images, however, they are omitted for simplicity in Table 2.

The number of the highest values indicated in boldface in Table 2 for each method is shown in Fig. 8, where the 


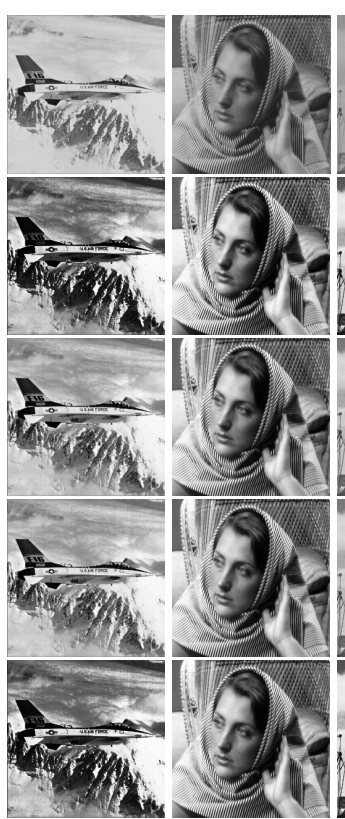

Airplane

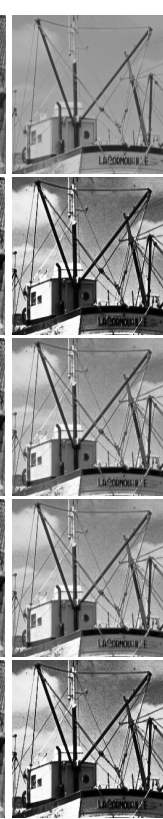

Boat

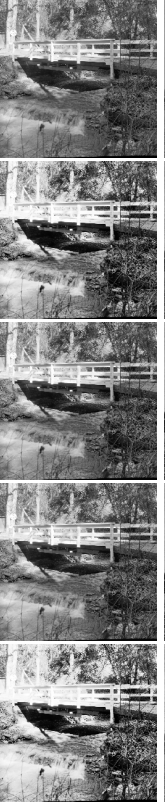

Bridge

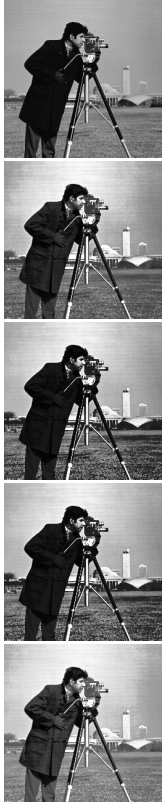

Cameraman



Lax

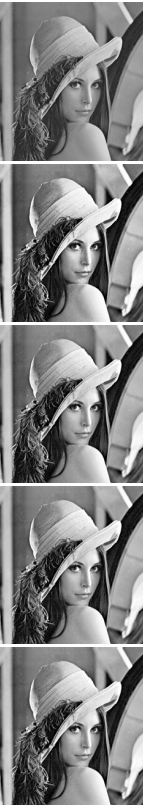

Lena

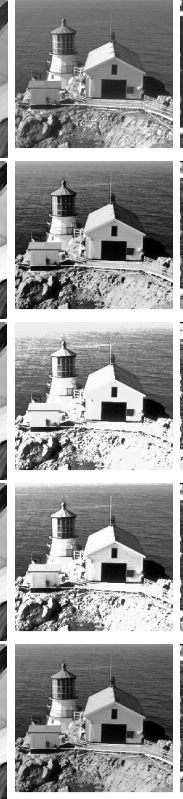

Lighthouse

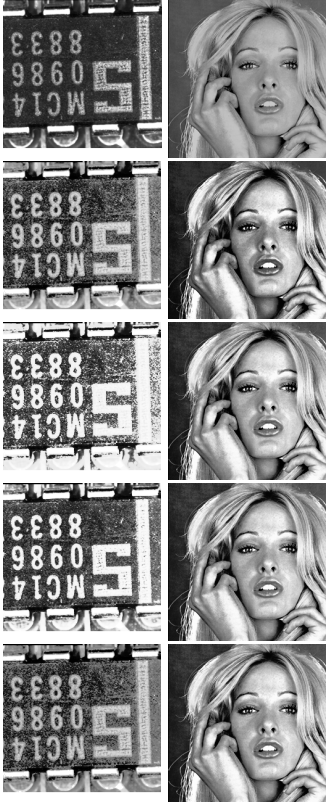

Text
Woman

Figure 7: Contrast enhancement results. The top row shows the original images from SIDBA [7], and the second to fifth rows show the output images by HE, piecewise-constant, -linear interpolations and local min-max interpolation, respectively.

Table 2: The values of image quality measures.

\begin{tabular}{|c|c|c|c|c|c|c|c|c|c|c|c|c|}
\hline \multirow[t]{2}{*}{ Images } & \multicolumn{4}{|c|}{ EMEG } & \multicolumn{4}{|c|}{ GMSD } & \multicolumn{4}{|c|}{$\mathrm{E} / \mathrm{G}$} \\
\hline & $\mathrm{HE}$ & Const. & Linear & Min-max & $\mathrm{HE}$ & Const. & Linear & Min-max & $\mathrm{HE}$ & Const. & Linear & Min-max \\
\hline Airplane & 0.3782 & 0.2611 & 0.2618 & 0.3834 & 0.2079 & 0.1149 & 0.1169 & 0.2353 & 1.8191 & 2.2727 & 2.2403 & 1.6292 \\
\hline Barbara & 0.2740 & 0.255 & 0.255 & 0.2547 & .0404 & 0.0221 & 0.0222 & 0.0228 & 6.7873 & 11.540 & 11.48 & 11.181 \\
\hline Boat & 4182 & 0.2804 & 0.2818 & 0.4394 & 0.2119 & 0.1356 & 0.1367 & 0.2336 & 1.9736 & 2.0669 & 2.0618 & 1.8809 \\
\hline Brid & 612 & 0.3704 & 0.3 & 5110 & 56 & 12 & 0.0096 & 96 & .2985 & 33.155 & 38.7646 & 5.1308 \\
\hline Building & 3194 & 0.3693 & 0.3705 & 0.2702 & .0738 & 0.1499 & 0.1734 & 0.0440 & 4.3281 & 2.4628 & 2.1368 & 6.1473 \\
\hline Camerama & 0.2808 & 0.3111 & 0.3130 & 0.2592 & .1167 & 0.1140 & 0.1150 & $\overline{0.1519}$ & 2.4069 & 2.7301 & 2.7216 & $\overline{1.7067}$ \\
\hline Girl & 0.2466 & 0.1938 & 0.1939 & 0.2628 & 1409 & 0.0602 & 0.059 & & 1.7497 & 3.2189 & 3.2846 & 1.4947 \\
\hline $\mathrm{L}$ & 0.6473 & 0.5 & 0.5 & & & & 69 & & 3.9986 & 901 & & 3.2768 \\
\hline Lena & 537 & 0.2295 & 0.2295 & 0.2317 & 0.0650 & 0.0330 & .0332 & 0.0348 & 3.9062 & 6.9466 & 6.9102 & 6.6646 \\
\hline Lighthouse & 3848 & 0.4220 & 0.4372 & 0.3234 & 0.090 & 0.1727 & 0.2189 & $\underline{0.0413}$ & 4.2736 & 2.4432 & 1.9978 & $\underline{7.8330}$ \\
\hline Text & 0.4101 & 0.5037 & 0.6053 & 0.3901 & 0.1716 & 0.1748 & 0.2636 & $\overline{0.1860}$ & 2.3896 & 2.8814 & 2.2965 & $\overline{2.0969}$ \\
\hline Woman & 0.2869 & 0.2690 & 0.2721 & 0.2692 & 0.1171 & 0.0692 & 0.0704 & 0.0699 & 2.4506 & 3.8886 & 3.8655 & 3.8530 \\
\hline Average & 0.3634 & 0.3357 & 0.3464 & 0.3514 & 0.1211 & 0.0937 & 0.107 & 0.1237 & 3.6985 & 6.8330 & 7.1833 & 4.4079 \\
\hline
\end{tabular}

vertical and horizontal axes denote the number of the highest values and the compared methods, respectively. The proposed three histogram interpolation methods achieved larger numbers in the vertical axis than HE. This result demonstrates the effectiveness of the proposed histogram interpolation methods.

There can be several methods for selecting one of the three proposed histogram interpolation methods. For example, one candidate is to select one of the three methods interactively on the basis of the shape of the equalized histogram of an input image. If there are big impulsive elements and/or big gaps in the equalized histogram, then we will select the local min-max interpolation to avoid the contrast over-enhancement, or else we will select the piecewiseconstant or -linear interpolation. Another automatic method is to enhance an input image with the three methods to obtain three enhanced images, and then select the one which achieves the best value of an adopted image quality measure.

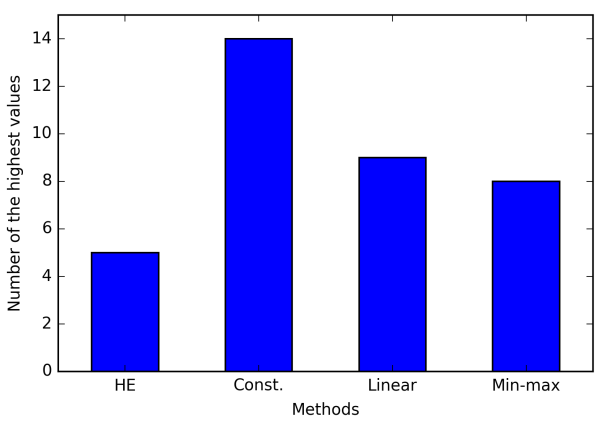

Figure 8: Number of the highest values in Table 2.

\section{Conclusion}

In this paper, we proposed three histogram interpolation methods for over-enhancement-suppressing image contrast enhancement, and showed that the interpolated histogram of the equalized histogram of a grayscale image can be used 
for the target histogram of histogram specification method for appropriate image contrast enhancement. Experimental results demonstrated the effectiveness of the proposed methods visually and numerically.

In the future work, we would like to apply the proposed methods to color image contrast enhancement incorporating gamut problems.

\section{Acknowledgment}

This work was supported by JSPS KAKENHI Grant Number JP16H03019.

\section{References}

[1] R. C. Gonzalez and R. E. Woods: "Digital Image Processing”, Pearson Prentice Hall, 3rd ed., 2008

[2] R. P. Singh and M. Dixit: "Histogram Equalization: A Strong Technique for Image Enhancement", International Journal of Signal Processing, Image Processing and Pattern Recognition, Vol. 8, No. 8, pp. 345-352, 2015. DOI: 10.14257/ijsip.2015.8.8.35

[3] H. Yu, K. Inoue, K. Hara, and K. Urahama: "Image Contrast Enhancement by Stepwise Histogram Specification”, Journal of Institute of Industrial Applications Engineers, Vol. 3, No. 4, pp. 161-166, 2015. DOI: 10.12792/JIIAE.2.161

[4] M. Nikolova and G. Steidl: "Fast Ordering Algorithm for Exact Histogram Specification", IEEE Trans. Image Process., Vol. 23, No. 12, pp. 5274-5283, 2014. DOI: 10.1109/TIP.2014.2364119

[5] T. Celik: "Spatial Entropy-Based Global and Local Image Contrast Enhancement", IEEE Trans. Image Process., Vol. 23, No. 12, pp. 5298-5308, 2014. DOI: 10.1109/TIP.2014.2364537

[6] W. Xue, L. Zhang, X. Mou, and A. C. Bovik: "Gradient Magnitude Similarity Deviation: A Highly Efficient Perceptual Image Quality Index", IEEE Trans. Image Process., Vol. 23, No. 2, pp. 684-695, 2014. DOI: 10.1109/TIP.2013.2293423

[7] M. Sakauchi, Y. Ohsawa, M. Sone, and M. Onoe: "Management of the Standard Image Database for Image Processing Researches", ITEJ Technical Report, Vol. 8, No. 38, pp. 712, 1984 (in Japanese)

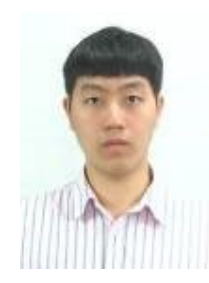

Hengjun Yu(Member) He received B.Eng. degree from University of Electronic Science and Technology of China in 2014. He is currently a graduate student in Kyushu University. His research interests include image processing and gesture recognition.

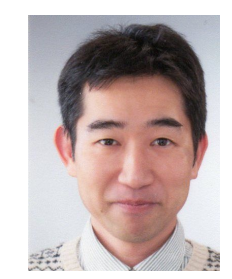

Kohei Inoue (Member) He received B.Des., M.Des. and D.Eng. degrees from Kyushu Institute of Design in 1996, 1998 and 2000, respectively. $\mathrm{He}$ is currently an Associate Professor in Kyushu University. His research interests include pattern recognition and image processing.

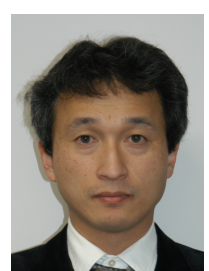

Kenji Hara (Non-member) He received the $\mathrm{BE}$ and ME degrees from Kyoto University in 1987 and 1989, respectively, and the $\mathrm{PhD}$ degree from Kyushu University in 1999. He is currently an Associate Professor in Kyushu University. His research interests include physics-based vision and geometric modeling.

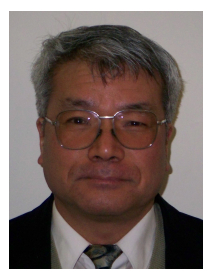

Kiichi Urahama (Member) $\mathrm{He}$ received M.Eng. and D.Eng. degrees from Kyushu University in 1976 and 1980 . From 1980 to 1995 he was an Associate Professor in Kyushu Institute of Technology. He is now a Professor in Kyushu University. His research interests include pattern recognition, image processing and computer graphics. 